\title{
Mean-Square Radius of Gyration and Second Virial Coefficient of Poly(diisopropyl fumarate) in Dilute Solution
}

\author{
By Masayuki NAKATSUJI, Munehiro HYAKUTAKE, Masashi OSA, and Takenao YoSHIZAKI*
}

The mean-square radius of gyration $\left\langle S^{2}\right\rangle$ and second virial coefficient $A_{2}$ were determined from light scattering measurements for 13 samples of poly(diisopropyl fumarate) (PDiPF), each with the fraction of racemo diads $f_{\mathrm{r}}=0.22$, in a range of weightaverage molecular weight from $4.02 \times 10^{4}$ to $8.59 \times 10^{5}$ in tetrahydrofuran at $30.0^{\circ} \mathrm{C}$. From a simultaneous analysis of the present data for $\left\langle S^{2}\right\rangle$ and $A_{2}$ on the basis of the Kratky-Porod (KP) wormlike chain with excluded volume, the KP model parameters for PDiPF, i.e., the stiffness parameter $\lambda^{-1}$ and the shift factor $M_{\mathrm{L}}$ are determined to be $113 \AA$ and $89 \AA^{-1}$, respectively, the $M_{\mathrm{L}}$ value being consistent with that estimated on the basis of chain conformations of PDiPF. The $\lambda^{-1}$ value indicates that PDiPF is stiffer than typical flexible polymers but not so stiff as typical semiflexible polymers such as poly $(n-$ hexyl isocyanate).

KEY WORDS: Poly(diisopropyl fumarate) / Mean-Square Radius of Gyration / Second Virial Coefficient / Semiflexible Polymer /

Kratky-Porod Wormlike Chain / Excluded-Volume Effect /

About 30 years ago, Bengough et al. ${ }^{1}$ and Otsu et al. $^{2-5}$ found that dialkyl fumarate (DRF) such as isopropyl fumarate can polymerize to yield poly(DRF) through radical polymerization. Because of steric hindrances to internal rotations caused by substituents on every main-chain carbon atom, poly(DRF) chains were considered to be stiffer than familiar vinyl polymer chains having substituents on every other mainchain carbon atom. Subsequently, Matsumoto and Nakagawa ${ }^{6}$ confirmed the conjecture by an analysis of experimental data for the intrinsic viscosity of poly(diisopropyl fumarate) (PDiPF) in tetrahydrofuran (THF) at $30.0^{\circ} \mathrm{C}$ on the basis of the Kratky-Porod (KP) wormlike chain model. ${ }^{7,8}$ The value of the stiffness parameter $\lambda^{-1}$ for PDiPF was determined to be $220 \AA$, which is appreciably larger than those for typical flexible polymers such as atactic polystyrene (a-PS) $\left(\lambda^{-1}=\right.$ $20.6 \AA)^{8,9}$ or atactic poly(methyl methacrylate) (a-PMMA) $\left(\lambda^{-1}=57.9 \AA\right){ }^{8,10}$

In the above analysis, Matsumoto and Nakagawa assumed that the intramolecular excluded-volume effect was negligibly small if any, although THF is a good solvent for PDiPF. The effect may actually be ignored for a semiflexible polymer if its $\lambda^{-1}$ is very large and its molecular weight $M$ (chain length) is not very large, as in the case of poly ( $n$-hexyl isocyanate) (PHIC) whose $\lambda^{-1}$ is $840 \AA \AA^{11}$ The $\lambda^{-1}$ value $220 \AA$ determined for PDiPF is, however, not so large as that for PHIC. Further, in a recent study of dilute solution properties of cellulose tris(phenyl carbamate) (CTC) made by Kasabo et al., ${ }^{12}$ it has been shown that the intramolecular excluded-volume effect cannot be ignored even for a polymer with $\lambda^{-1}=210 \AA$ in an ordinary range of $M$. In order to evaluate $\lambda^{-1}$ accurately for PDiPF, therefore, it is desirable to make an analysis with due consideration of the excluded-volume effects. This is the purpose of the present paper.
The determination of $\lambda^{-1}$ for PDiPF in a good solvent requires some comments. We could determine it from an analysis only of the mean-square radius of gyration $\left\langle S^{2}\right\rangle$ as a function of the weight-average molecular weight $M_{\mathrm{w}}$ by the use of the method proposed by Murakami et al., ${ }^{11}$ if experimental data were obtained for $\left\langle S^{2}\right\rangle$ in an $M_{\mathrm{w}}$ range where the intramolecular excluded-volume effect might be ignored. In anticipation of results, we note that the effect on $\left\langle S^{2}\right\rangle$ cannot be ignored in the whole range of $M_{\mathrm{w}}\left(4.02 \times 10^{4} \leq M_{\mathrm{w}} \leq 8.59 \times\right.$ $10^{5}$ ) examined. Then we make a maiden attempt to analyze $\left\langle S^{2}\right\rangle$ and the second virial coefficient $A_{2}$ simultaneously, both determined from light scattering (LS) measurements, on the

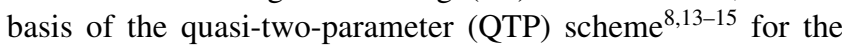
intramolecular excluded-volume effect and also the Yamakawa theory ${ }^{8,16}$ of $A_{2}$ which is concerned with the intermolecular one.

\section{EXPERIMENTAL}

\section{Materials}

Three original PDiPF samples were synthesized by radical polymerization, following the procedure of Otsu et al. ${ }^{4,5}$ and Matsumoto and Nakagawa. ${ }^{6}$ Polymerization was carried out in benzene by the use of dimethyl $2,2^{\prime}$-azobis(isobutyrate) as an initiator under dry nitrogen at $60^{\circ} \mathrm{C}$ for $c a .120 \mathrm{~h}$ for two samples in the range of $M_{\mathrm{w}} \lesssim 4 \times 10^{5}$ and under a high vacuum condition at $40{ }^{\circ} \mathrm{C}$ for $\mathrm{ca}$. $240 \mathrm{~h}$ for the other sample having larger $M_{\mathrm{w}}$. It was terminated by pouring each polymerization mixture into a large amount of hexane to precipitate an original sample. Each original sample so synthesized was separated into fractions by fractional precipitation using benzene as a solvent and methanol as a precipitant. 13 test samples so obtained were freeze-dried from their benzene solutions after filtration through a Teflon membrane of pore size $1.0 \mu \mathrm{m}$. 
The values of the ratio of $M_{\mathrm{w}}$ to the number-average molecular weight $M_{\mathrm{n}}$ for all the samples except the two having the smallest and largest $M_{\mathrm{w}}$ were determined from analytical gel permeation chromatography (GPC) using tetrahydrofuran as an eluent.

The solvent THF used for LS was purified by distillation after refluxing over sodium. The solvents THF and deuterated chloroform used for analytical GPC and ${ }^{13} \mathrm{C}$ NMR spectroscopy, respectively, were of reagent grade.

\section{Light Scattering}

LS measurements were carried out to determine $M_{\mathrm{w}}$ and $A_{2}$ for all the 13 test samples and $\left\langle S^{2}\right\rangle$ for 10 test samples with $M_{\mathrm{w}} \gtrsim 8 \times 10^{4}$, in THF at $30.0^{\circ} \mathrm{C}$. A Fica 50 light-scattering photometer was used for all the measurements with vertically polarized incident light of wavelength $\lambda_{0}=436 \mathrm{~nm}$. For a calibration of the apparatus, the intensity of light scattered from pure benzene was measured at $25.0^{\circ} \mathrm{C}$ at a scattering angle of $90^{\circ}$, where the Rayleigh ratio $R_{\mathrm{Uu}}\left(90^{\circ}\right)$ of pure benzene was taken as $46.5 \times 10^{-6} \mathrm{~cm}^{-1} .{ }^{17}$ The depolarization ratio $\rho_{\mathrm{u}}$ of pure benzene at $25.0^{\circ} \mathrm{C}$ was determined to be $0.41 \pm 0.01$. Scattered intensity was measured at six different concentrations and at scattering angles $\theta$ ranging from 30.0 to $142.5^{\circ}$. The data obtained were treated by using the Berry square-root plot. ${ }^{18}$ For three samples with $M_{\mathrm{w}} \lesssim 7 \times 10^{4}$, corrections for optical anisotropy were necessary to estimate the true $M_{\mathrm{w}}$ and $A_{2}$. Therefore, the excess depolarized (Hv) components $\Delta R_{\mathrm{Hv}}$ of the reduced scattered intensity for their solutions were measured in addition to the excess unpolarized (Uv) components $\Delta R_{\mathrm{Uv}}$ in order to make those necessary corrections. As for the other samples with $M_{\mathrm{w}} \gtrsim 8 \times 10^{4}$, effects of optical anisotropy were negligibly small, so that the corrections were unnecessary and only $\Delta R_{\mathrm{Uv}}$ was measured for each solution.

The most concentrated solution of each sample was prepared gravimetrically and made homogeneous by continuous stirring in the dark at room temperature for $2 \mathrm{~d}$. It was optically purified by filtration through a Teflon membrane of pore size 0.45 or $0.10 \mu \mathrm{m}$. The solutions of lower concentrations were obtained by successive dilution. The weight concentrations were converted to the polymer mass concentrations $c$ by the use of the densities of the respective solutions calculated with the partial specific volumes $v_{2}$ of the samples and with the density $\rho_{0}$ of the solvent THF. The quantities $v_{2}$ and $\rho_{0}$ were measured with a pycnometer of the LipkinDavison type having a volume of $3 \mathrm{~cm}^{3}$. The values of $v_{2}$ so determined in THF at $30.0^{\circ} \mathrm{C}$ are $0.873 \mathrm{~cm}^{3} / \mathrm{g}$ for all the samples independently of $M_{\mathrm{w}}$. The value of $\rho_{0}$ of THF at $30.0{ }^{\circ} \mathrm{C}$ is $0.8751 \mathrm{~g} / \mathrm{cm}^{3}$.

The refractive index increment $\partial n / \partial c$ was measured at the wavelength of $436 \mathrm{~nm}$ by the use of a Shimadzu differential refractometer. The values of $\partial n / \partial c$ so determined in THF at $30.0{ }^{\circ} \mathrm{C}$ are $0.0623_{3} \mathrm{~cm}^{3} / \mathrm{g}$ for all the samples independently of $M_{\mathrm{w}}$. The value of the refractive index $n_{0}$ of THF at $30.0^{\circ} \mathrm{C}$ and at the wavelength of $436 \mathrm{~nm}$ was determined to be $1.410_{8}$ by the use of an Abbe refractometer (ERMA OPTICAL WORKS).

\section{${ }^{13}$ C NMR}

${ }^{13} \mathrm{C}$ NMR spectra for three of the 13 test samples and for the (unfractionated) three original samples were recorded on a JEOL JNM EX-400 spectrometer at 100.4 MHz. The spectra were taken in deuterated chloroform at room temperature using an rf pulse angle of $90^{\circ}$ with a pulse repetition time of $3 \mathrm{~s}$. The relative chemical shifts $\delta_{\mathrm{r}}$ of ${ }^{13} \mathrm{C}$ signals of a test sample referred to the ${ }^{13} \mathrm{C}$ signal of chloroform were measured and then converted to the chemical shifts in the tetramethylsilane scale $\delta=\delta_{\mathrm{r}}+77.00 \mathrm{ppm}$.

\section{RESULTS}

As is well known, we cannot correctly evaluate $M_{\mathrm{w}},\left\langle S^{2}\right\rangle$, and $A_{2}$ from the (standard) Berry square-root plot if the effects of optical anisotropy of polymer chains are not small, ${ }^{19-23}$ and must make an appropriate correction for the optical anisotropy. Following the standard procedure ${ }^{19-23}$ by the use of the reduced Hv component $\Delta R_{\mathrm{Hv}}$ of the excess scattered intensity in addition to the Uv component $\Delta R_{\mathrm{Uv}}$ used for the square-root plot, we may calculate $M_{\mathrm{w}}$ and the optical anisotropy factor $\delta$ from

$$
\begin{aligned}
& \lim _{c, \theta \rightarrow 0} \frac{\Delta R_{\mathrm{Uv}}}{2 K c}=M_{\mathrm{w}, \mathrm{ap}}=(1+7 \delta) M_{\mathrm{w}} \\
& \lim _{c, \theta \rightarrow 0} \frac{\Delta R_{\mathrm{Hv}}}{2 K c}=3 \delta M_{\mathrm{w}}
\end{aligned}
$$

with the values of $\Delta R_{\mathrm{Uv}} / 2 K c$ and $\Delta R_{\mathrm{Hv}} / 2 K c$ in the limit of the vanishing mass concentration $c$ and scattering angle $\theta$. In eqs 1 and 2, $K$ is the optical constant and $M_{\mathrm{w}, \text { ap }}$ is the the apparent weight-average molecular weight determined directly from the square-root plot. In the second and third columns of Table I are given the values of $M_{\mathrm{w}}$ and $\delta$ so determined for all the 13 samples. We note that the correction for the optical anisotropy is not necessary for the samples PDiPF8 through PDiPF86.

For the three samples PDiPF4, PDiPF5, and PDiPF6, the apparent mean-square radius of gyration $\left\langle S^{2}\right\rangle_{\text {ap }}$ could not be evaluated directly from the square-root plot with sufficient accuracy to estimate (true) $\left\langle S^{2}\right\rangle$, because the slopes of the plots are very small for these samples. As for the other 10 samples with larger $M_{\mathrm{w}}$, for which correction for the optical anisotropy is unnecessary, $\left\langle S^{2}\right\rangle$ for each sample may be equated to $\left\langle S^{2}\right\rangle_{\text {ap }}$

Table I. Experimental Results for Poly(diisopropyl fumarate)

\begin{tabular}{lcccccc}
\hline sample & $M_{\mathrm{w}}$ & $10^{3} \delta$ & $\begin{array}{c}10^{-4}\left\langle S^{2}\right\rangle \\
\left(\AA^{2}\right)\end{array}$ & $\begin{array}{c}10^{4} A_{2} \\
\left(\mathrm{~cm}^{3} \mathrm{~mol} / \mathrm{g}^{2}\right)\end{array}$ & $M_{\mathrm{w}} / M_{\mathrm{n}}$ & $f_{\mathrm{r}}$ \\
\hline PDiPF4 & $4.02 \times 10^{4}$ & $9.1_{2}$ & & $5.1_{4}$ & & \\
PDiPF5 & $4.95 \times 10^{4}$ & $8.8_{6}$ & & $5.4_{5}$ & 1.10 & 0.22 \\
PDiPF6 & $6.31 \times 10^{4}$ & $5.2_{0}$ & & $4.9_{3}$ & 1.05 & \\
PDiPF8 & $8.00 \times 10^{4}$ & & $1.4_{2}$ & $4.2_{5}$ & 1.10 & \\
PDiPF9 & $9.17 \times 10^{4}$ & & $1.7_{9}$ & $4.0_{7}$ & 1.07 & \\
PDiPF15 & $1.53 \times 10^{5}$ & & $3.1_{9}$ & $4.0_{0}$ & 1.08 & \\
PDiPF18 & $1.83 \times 10^{5}$ & & $3.9_{1}$ & $3.5_{1}$ & 1.08 & 0.22 \\
PDiPF22 & $2.18 \times 10^{5}$ & & $5.0_{3}$ & $3.7_{5}$ & 1.09 & \\
PDiPF34 & $3.37 \times 10^{5}$ & & $7.9_{8}$ & $3.5_{3}$ & 1.07 & \\
PDiPF44 & $4.37 \times 10^{5}$ & 10.8 & $3.4_{5}$ & 1.07 & \\
PDiPF50 & $5.00 \times 10^{5}$ & 11.8 & $3.3_{4}$ & 1.05 & 0.22 \\
PDiPF62 & $6.20 \times 10^{5}$ & 15.3 & $2.9_{1}$ & 1.05 & \\
PDiPF86 & $8.59 \times 10^{5}$ & 20.9 & $2.9_{9}$ & & \\
\hline
\end{tabular}


evaluated directly from the slope of the plot. The values of $\left\langle S^{2}\right\rangle$ so determined for the 10 samples are given in the fourth column of Table I.

The correction for the optical anisotropy to $A_{2}$ may be given by ${ }^{19-21}$

$$
A_{2, \text { ap }}=A_{2}(1+7 \delta)^{-2}
$$

For the three samples PDiPF4, PDiPF5, and PDiPF6, the values of $A_{2}$ have been calculated from eq 3 with the values of $\delta$ given in the third column of Table I and also the values of the apparent second virial coefficient $A_{2 \text {,ap }}$ determined directly from the square-root plot. As for the other 10 samples with larger $M_{\mathrm{w}}$, for which the correction for the optical anisotropy is unnecessary, $A_{2}$ for each sample may be equated to $A_{2, \text { ap }}$. The values of $A_{2}$ so determined for all the samples are given in the fifth column of Table I.

In the sixth column of Table I are also given the values of the ratio of $M_{\mathrm{w}}$ to $M_{\mathrm{n}}$ determined for all the samples except PDiPF4 and PDiPF86 by analytical GPC with the use of the values of $M_{\mathrm{w}}$ of all the samples given in the second column of the table. It is seen that the samples PDiPF5 through PDiPF62 are very narrow in molecular weight distribution. Although $M_{\mathrm{w}} / M_{\mathrm{n}}$ could not be evaluated for the samples PDiPF4 and PDiPF86 with high accuracy because of lack of the calibration curve in the necessary range, their molecular weight distribution may be considered to be as narrow as that of the other samples.

From intensity of signals in the ${ }^{13} \mathrm{C}$ NMR spectra in a range of the chemical shift from 40.0 to $50.0 \mathrm{ppm}$, we have determined stereochemical compositions of the three samples PDiPF5, PDiPF18, and PDiPF50 and also of the (unfractionated) three original samples. Naturally, stereochemical terminology used for PDiPF composed of successive (pseudo-)asymmetric backbone carbon atoms is different from that for vinyl polymers in which every other backbone carbon atoms are asymmetric. Following the terminology proposed by Wang et al.,${ }^{24}$ we call a pair of adjacent backbone carbon atoms (diad) in a PDiPF chain meso if vicinal substituents joined to them are located on opposite sides of a plane containing all the backbone carbon atoms of the PDiPF chain in the planar trans conformation, and racemo otherwise. According to Wang et $a{ }^{24}$ and Yoshioka et al. ${ }^{25}$ the signals in the above range of the chemical shift may then be assigned to the meso and racemo diads. The stereochemical compositions specified by fractions $f_{\mathrm{r}}$ of racemo diads for the test samples PDiPF5, PDiPF18, and PDiPF50 are given in the last column of Table I, which are consistent with the literature values ${ }^{24,25} 0.22-0.23$. The values of $f_{\mathrm{r}}$ for the original samples are the same as those for the three test samples, indicating that all the PDiPF samples should have the same value 0.22 of $f_{\mathrm{r}}$.

Figure 1 shows double-logarithmic plots of $\left\langle S^{2}\right\rangle / M_{\mathrm{w}}\left(\left\langle S^{2}\right\rangle\right.$ in $\AA^{2}$ ) against $M_{\mathrm{w}}$ for PDiPF in THF at $30.0^{\circ} \mathrm{C}$. The unfilled circles represent the present experimental data. The solid and dashed curves represent the theoretical values for the KP chain with and without excluded volume, respectively, which are obtained and discussed in the next (DISCUSSION) section. It is seen that $\left\langle S^{2}\right\rangle / M_{\mathrm{w}}$ increases monotonically with increasing

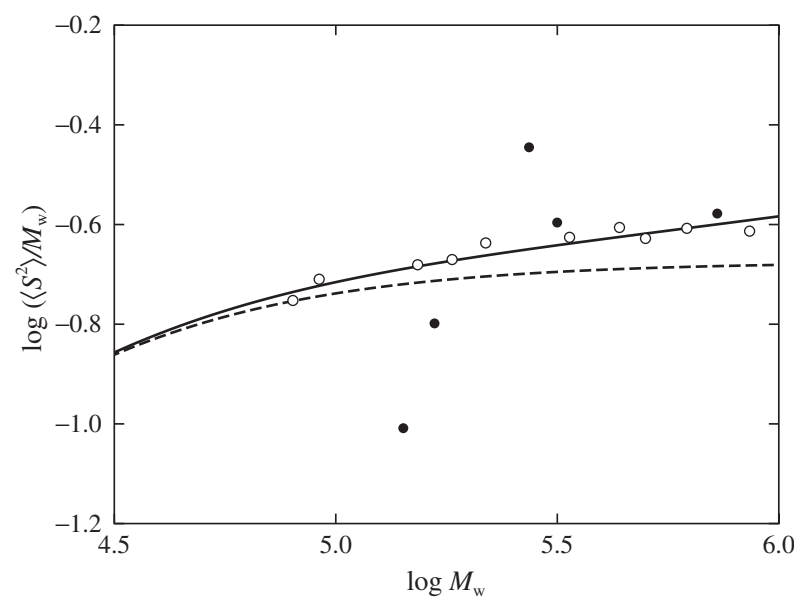

Figure 1. Double-logarithmic plots of $\left\langle S^{2}\right\rangle / M_{\mathrm{w}}\left(\left\langle S^{2}\right\rangle\right.$ in $\left.\AA^{2}\right)$ against $M_{\mathrm{w}}$ for PDiPF in THF at $30.0^{\circ} \mathrm{C}$ : (O) present data; (O) data by Matsumoto and Nakagawa. ${ }^{6}$ The solid and dashed curves represent the best-fit theoretical values for the KP chain with and without excluded volume.

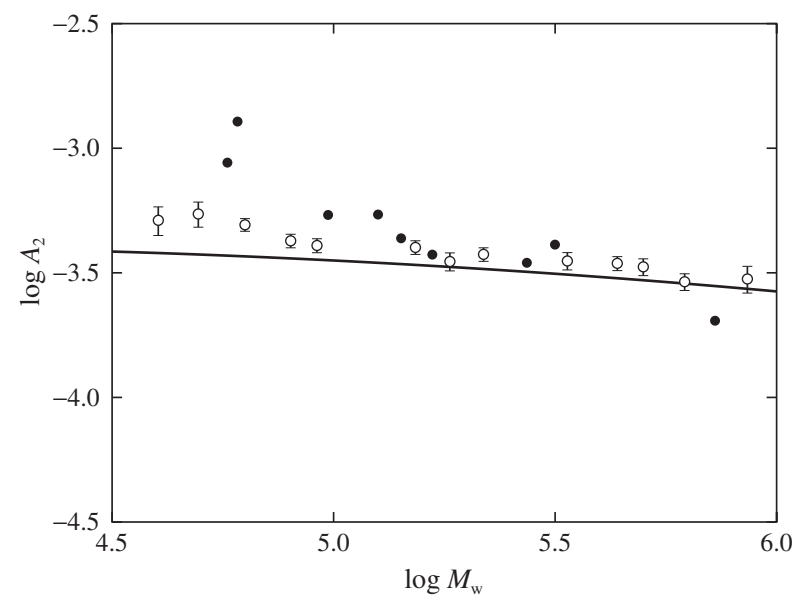

Figure 2. Double-logarithmic plots of $A_{2}$ (in $\mathrm{cm}^{3} \mathrm{~mol} / \mathrm{g}^{2}$ ) against $M_{\mathrm{w}}$ for PDiPF in THF at $30.0^{\circ} \mathrm{C}$. All the symbols have the same meaning as those in Figure 1. The vertical line segments attached to the present data points $(\bigcirc)$ indicate the error bounds. The solid curve represents the theoretical values for the KP chain without the effects of chain ends (see the text).

$M_{\mathrm{w}}$. It is also seen that the present data points form a curve convex upward, the slope of which is $c a$. 0.19 for $M_{\mathrm{w}} \lesssim$ $2 \times 10^{5}$ and $c a$. 0.08 for $M_{\mathrm{w}} \gtrsim 3 \times 10^{5}$. The rather steep slope in the range of $M_{\mathrm{w}} \lesssim 2 \times 10^{5}$ may be due to effects of chain stiffness of PDiPF. For comparison, literature data for PDiPF in THF at $30.0^{\circ} \mathrm{C}$ by Matsumoto and Nakagawa ${ }^{6}$ are also plotted in the figure (filled circles). Their values are scattered around ours in the range of $M_{\mathrm{w}} \lesssim 3 \times 10^{5}$ but agree with ours within experimental error in the range of $M_{\mathrm{w}} \gtrsim 3 \times 10^{5}$.

Figure 2 shows double-logarithmic plots of $A_{2}$ (in $\mathrm{cm}^{3} \mathrm{~mol} /$ $\left.\mathrm{g}^{2}\right)$ against $M_{\mathrm{w}}$ for PDiPF in THF at $30.0^{\circ} \mathrm{C}$. All the symbols have the same meaning as those in Figure 1. The vertical line segment attached to each present data point $(O)$ indicates its error bound. The solid curve represents the theoretical values for the KP chain without effects of chain ends, which are 
obtained and discussed in the next (DISCUSSION) section. The present data points follow a straight line of slope $c a$. -0.13 , which is appreciably larger than $c a$. -0.2 for typical flexible polymers with large $M_{\mathrm{w}}$ in good solvents, implying that the PDiPF chain has rather large stiffness. The literature data obtained by Matsumoto and Nakagawa ${ }^{6}$ (filled circles) agree well with the present ones, although they are somewhat scattered in the range of $M_{\mathrm{w}} \lesssim 1 \times 10^{5}$.

\section{DISCUSSION}

Now we proceed to analyze the present data for $\left\langle S^{2}\right\rangle$ and $A_{2}$ for PDiPF given in the last section on the basis of the KP theory of the unperturbed mean-square radius of gyration ${ }^{8,26}\left\langle S^{2}\right\rangle_{0}$ along with the QTP scheme or the Yamakawa-StockmayerShimada theory ${ }^{8,13-15}$ of the intramolecular excluded-volume effect and also of the Yamakawa theory ${ }^{8,16}$ of $A_{2}$.

For the KP chain of total contour length $L,\left\langle S^{2}\right\rangle$ may be written in the form,

$$
\left\langle S^{2}\right\rangle=\left\langle S^{2}\right\rangle_{0} \alpha_{S}^{2}
$$

where $\left\langle S^{2}\right\rangle_{0}$ is given by ${ }^{8,26}$

$$
\left\langle S^{2}\right\rangle_{0}=\frac{L}{6 \lambda}-\frac{1}{4 \lambda^{2}}+\frac{1}{4 \lambda^{3} L}-\frac{1}{8 \lambda^{4} L^{2}}\left(1-\mathrm{e}^{-2 \lambda L}\right)
$$

and the gyration-radius expansion factor $\alpha_{S}$ may be given by the Domb-Barrett equation, ${ }^{27}$

$$
\begin{aligned}
\alpha_{S}^{2}= & {\left[1+10 \tilde{z}+\left(\frac{70 \pi}{9}+\frac{10}{3}\right) \tilde{z}^{2}+8 \pi^{3 / 2} \tilde{z}^{3}\right]^{2 / 15} } \\
& \times\left[0.933+0.067 \exp \left(-0.85 \tilde{z}-1.39 \tilde{z}^{2}\right)\right]
\end{aligned}
$$

In the QTP scheme, the (conventional) excluded-volume parameter $z$ defined in the two-parameter (TP) scheme on the basis of the Gaussian chain model is replaced by the intramolecular scaled excluded-volume parameter $\tilde{z}$ defined by

$$
\tilde{z}=\frac{3}{4} K(\lambda L) z
$$

where the coefficient $K(\lambda L)$ as a function of the reduced contour length $\lambda L$ is given by

$$
\begin{aligned}
K(\lambda L)= & \frac{4}{3}-2.711(\lambda L)^{-1 / 2}+\frac{7}{6}(\lambda L)^{-1} & & \text { for } \lambda L>6 \\
= & (\lambda L)^{-1 / 2} \exp \left[-6.611(\lambda L)^{-1}\right. & & \\
& +0.9198+0.03516 \lambda L] & & \text { for } \lambda L \leq 6
\end{aligned}
$$

The quantity $K(\lambda L)$ represents the effects of chain stiffness on the intramolecular excluded-volume effect or on the intramolecular contact probability and increases monotonically from 0 to $4 / 3$ as $\lambda L$ is increased from 0 to $\infty$. Then, in the limit of $\lambda L \rightarrow \infty$ (random-coil limit) the QTP scheme reduces to the
TP one, i.e., $\tilde{z}=z$, while in the limit of $\lambda L \rightarrow 0$ (rod limit) $\tilde{z}$ vanishes irrespective of the value of $z$.

The QTP scheme is formulated on the basis of a model composed of $n+1$ beads of the binary-cluster integral $\beta$ arrayed with spacing $a$ between them along the KP or helical wormlike (HW) chain ${ }^{8}$ of total contour length $L$, so that $L=n a$. The parameter $z$ may then be written in terms of $\beta, a$, and $\lambda L$ as

$$
z=(3 / 2 \pi)^{3 / 2}(\lambda B)(\lambda L)^{1 / 2}
$$

where $B$ is the excluded-volume strength defined by

$$
B=\beta / a^{2} c_{\infty}^{3 / 2}
$$

with

$$
c_{\infty}=\lim _{\lambda L \rightarrow \infty}\left(6 \lambda\left\langle S^{2}\right\rangle_{0} / L\right)
$$

As seen from eq 11 with eq $5, c_{\infty}$ is equal to unity for the KP chain.

In the Yamakawa theory ${ }^{8,16}$ of $A_{2}$, effects of chain stiffness and chain ends are taken into account, and $A_{2}$ may then be written in the form,

$$
A_{2}=A_{2}^{(\mathrm{HW})}+A_{2}^{(\mathrm{E})}
$$

where $A_{2}^{(\mathrm{HW})}$ is a part of $A_{2}$ without the effects of chain ends and $A_{2}^{(\mathrm{E})}$ represents contribution of the effects of chain ends to $A_{2}$. The superscript (HW) attached to the first term indicates that the theory is formulated on the basis of the HW chain, and the following theoretical expression for $A_{2}^{(\mathrm{HW})}$ reduces to that for the KP chain if $c_{\infty}$ is set equal to unity.

For the KP (or HW) chain of total contour length $L$ with the excluded-volume strength $B, A_{2}^{(\mathrm{HW})}$ may be given by

$$
A_{2}^{(\mathrm{HW})}=\left(N_{\mathrm{A}} c_{\infty}^{3 / 2} L^{2} B / 2 M^{2}\right) h(\hat{z})
$$

where $N_{\mathrm{A}}$ is the Avogadro constant, $M$ is the polymer molecular weight, and $h$ is given by

$$
h(\hat{z})=\left(1+7.74 \hat{z}+52.3 \hat{z}^{27 / 10}\right)^{-10 / 27}
$$

with

$$
\hat{z}=\tilde{z} / \alpha_{S}^{3}
$$

In eq $15, \tilde{z}$ is the intermolecular scaled excluded-volume parameter defined by

$$
\tilde{\tilde{z}}=\left[\frac{Q(\lambda L)}{2.865}\right] z
$$

The coefficient $Q(\lambda L)$ in eq 16 as a function of $\lambda L$ represents the effects of chain stiffness on the intermolecular excludedvolume effect and is given in a very good approximation for $\lambda L \gtrsim 1$ by $^{8,16}$

$$
\begin{aligned}
Q(\lambda L)= & -\frac{128 \sqrt{2}}{15}-2.531(\lambda L)^{-1 / 2}-2.586(\lambda L)^{-1}+1.985(\lambda L)^{-3 / 2} \\
& -1.984(\lambda L)^{-2}-0.9292(\lambda L)^{-5 / 2}+0.1223(\lambda L)^{-3}+\frac{8}{5} x^{5 / 2} \\
& +\frac{2}{3} x^{3 / 2}\left[8+\frac{1}{6}(\lambda L)^{-1}\right]+x^{1 / 2}\left[8-13.53(\lambda L)^{-1}+0.2804(\lambda L)^{-2}\right] \\
& -x^{-1 / 2}(\lambda L)^{-1}\left[0.3333-5.724(\lambda L)^{-1}+0.7974(\lambda L)^{-2}\right] \\
& -x^{-3 / 2}(\lambda L)^{-2}\left[0.3398-0.7146(\lambda L)^{-1}\right]
\end{aligned}
$$


with

$$
x=1+0.961(\lambda L)^{-1}
$$

It is seen from eq 16 with eqs 17 and 18 that $\tilde{z}$ becomes identical with $z$ in the limit of $\lambda L \rightarrow \infty$ as in the case of $\tilde{z}$ and therefore $A_{2}^{(\mathrm{HW})}$ reduces to $A_{2}$ given by the TP theory.

As for the second term $A_{2}^{(\mathrm{E})}$ in eq 12 , we omit its detailed expression $^{8,16}$ since it is not necessary for the present analysis of $A_{2}$. We only note that $A_{2}^{(\mathrm{E})}$ rapidly decreases to 0 with increasing $M$ and may be neglected for $M \gtrsim 10^{5}$ for all the polymer-solvent systems examined so far. ${ }^{8,12,28-31}$

In order to compare the above-mentioned KP theoretical values of $\left\langle S^{2}\right\rangle$ and $A_{2}$ as functions of $L$ with the experimental ones as functions of $M, L$ is related to $M$ by

$$
L=M / M_{\mathrm{L}}
$$

where $M_{\mathrm{L}}$ is the shift factor as defined as the molecular weight per unit contour length of the KP (or HW) chain. We may then determine the three parameters $\lambda^{-1}, M_{\mathrm{L}}$, and $\lambda B$ by the curve fitting to the experimental data for $\left\langle S^{2}\right\rangle$ or $A_{2}$. As already mentioned, we cannot determine them only from the data for $\left\langle S^{2}\right\rangle$, so that we attempt to carry out the curve fitting to both the data for $\left\langle S^{2}\right\rangle$ and $A_{2}$ simultaneously.

In Figures 1 and 2, the solid curves represent the best-fit KP theory values of $\left\langle S^{2}\right\rangle$ and those of $A_{2}$ without consideration of the effects of chain ends $\left(A_{2}^{(\mathrm{E})}=0\right)$, respectively, where the former values have been calculated from eq 4 with eqs 5-9 and 19 and the latter from eq 12 with eqs 6-9 and 13-19, with $\lambda^{-1}=113 \AA, M_{\mathrm{L}}=89 \AA^{-1}$, and $\lambda B=0.097$. We note that relative errors in those parameter values are 8,5 , and $10 \%$, respectively, at most. It is seen from the figures that the theoretical values of $\left\langle S^{2}\right\rangle$ quantitatively reproduce the experimental ones over the whole range of $M_{\mathrm{w}}$ examined and the theoretical values of $A_{2}\left(=A_{2}^{(\mathrm{HW})}\right)$ may rather well reproduce the experimental ones within experimental error in the range of $M_{\mathrm{w}} \gtrsim 10^{5}$, where the effects of chain ends may be neglected. In Figure 2, the present data points deviate upward progressively from the theoretical curve with decreasing $M_{\mathrm{w}}$ for $M_{\mathrm{w}} \lesssim 10^{5}$. It may be regarded as arising from the effects of chain ends $\left(A_{2}^{(\mathrm{E})}=0\right)$. From an analysis of the difference between the observed $A_{2}$ and the theoretical $A_{2}^{(\mathrm{HW})}$, we may obtain information about the excess (effective) binary-cluster integrals of terminal beads. ${ }^{8,16}$ Such an analysis is beyond the scope of the present work, and we do not pursue further it.

In Figure 1 are also shown the theoretical values of $\left\langle S^{2}\right\rangle_{0}$ (dashed curve) calculated from eq 5 with the values of $\lambda^{-1}$ and $M_{\mathrm{L}}$ determined above. It is seen that the present data points deviate upward progressively from the theoretical $\left\langle S^{2}\right\rangle_{0}$ with increasing $M_{\mathrm{w}}$ due to the intramolecular excluded-volume effect.

Finally, it is interesting to analyze the present data for $\left\langle S^{2}\right\rangle$ shown in Figure 1 on the basis of the KP chain without excluded volume, as considered by Matsumoto and Nakagawa. ${ }^{6}$ We have obtained $\lambda^{-1}=154 \AA$ and $M_{\mathrm{L}}=103$ $\AA^{-1}$ from the curve fitting, although the best-fit unperturbed KP values of $\left\langle S^{2}\right\rangle_{0}$ have been omitted in Figure 1, for simplicity.

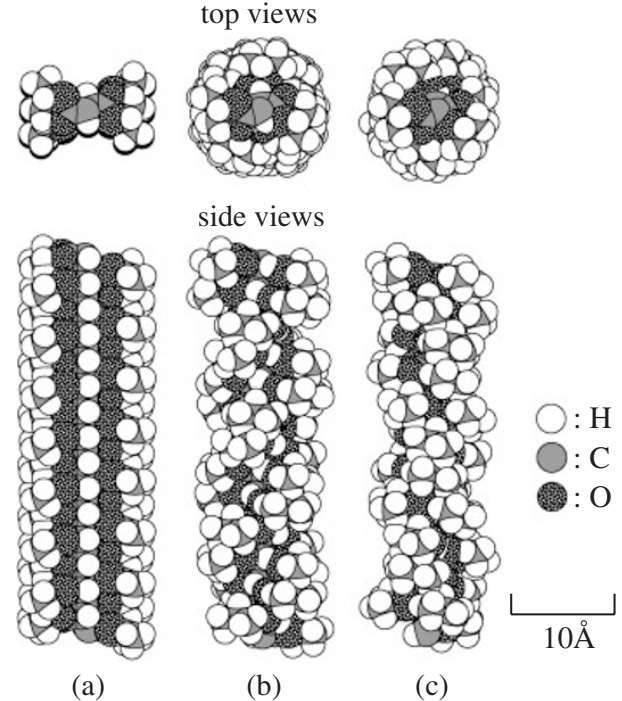

Figure 3. Schematic drawing of chain conformations of PDiPF with $f_{r}=0$, where all atoms are depicted by the spheres having the van der Walls radii: (a) planar trans conformation with $\phi=0$; (b) helical conformation with $\phi=30^{\circ}$; (c) helical conformation with $\phi=60^{\circ}$.

Note that the KP chain without excluded volume cannot explain the experimental data for $A_{2}$, needless to say. The value $89 \AA^{-1}$ of $M_{\mathrm{L}}$ determined from the present analysis with consideration of the intra- and intermolecular excluded-volume effects is remarkably smaller than the above value $103 \AA^{-1}$ and also the literature value $134 \AA^{-1}$ by Matsumoto and Nakagawa, both without consideration of the intramolecular excludedvolume effects.

For the PDiPF chain (with $f_{\mathrm{r}}=0$ ) in the planar trans conformation (with a bond angle of $109^{\circ}$ ), which is schematically depicted in Figure 3(a), $M_{\mathrm{L}}$ may be evaluated to be $80.3 \AA^{-1}$. A polymer chain in a helical conformation in principle has an $M_{\mathrm{L}}$ value larger than that for the chain in the planar trans conformation, and therefore the (true) $M_{\mathrm{L}}$ value for the PDiPF chain seems larger than $80.3 \AA^{-1}$. For trial calculations of $M_{\mathrm{L}}$ for the PDiPF chain (with $f_{\mathrm{r}}=0$ ) in helical conformations, we consider two chains having fixed internal rotation angles $\phi=30^{\circ}$ and $60^{\circ}$, which are schematically depicted in Figure 3(b) and 3(c), respectively. Note that the chain with $\phi=0^{\circ}$ is in the planar trans conformation. The values of $M_{\mathrm{L}}$ are evaluated to be 81.3 and $84.7 \AA^{-1}$ for $\phi=30^{\circ}$ and $60^{\circ}$, respectively, which are not very larger than $80.3 \AA^{-1}$. Considering this fact, the value $89 \AA^{-1}$ of $M_{\mathrm{L}}$ seems reasonable, and analyses of data for PDiPF in THF at $30.0^{\circ} \mathrm{C}$ without consideration of the excluded-volume effects lead to overestimation of the value of $M_{\mathrm{L}}$ and also that of $\lambda^{-1}$.

\section{CONCLUDING REMARKS}

We have carried out LS measurements to determine $\left\langle S^{2}\right\rangle$ and $A_{2}$ for PDiPF in THF at $30.0^{\circ} \mathrm{C}$ and then determined its $\mathrm{KP}$ model parameters $\lambda^{-1}$ and $M_{\mathrm{L}}$ by analyzing the data so obtained on the basis of the corresponding KP (or HW) theories 
with consideration of the intra- and intermolecular excludedvolume effects. The value $113 \AA$ of $\lambda^{-1}$ determined in the present analysis is smaller than $220 \AA$ obtained by Matsumoto and Nakagawa ${ }^{6}$ from an analysis of $[\eta]$ without consideration of the intramolecular excluded-volume effect. However, the qualitative characteristic of PDiPF proposed by them is still valid, i.e., its chain stiffness is larger than that of typical flexible polymers such as a-PS and a-PMMA but not so large as that of typical semiflexible polymers such as PHIC. The value $89 \AA^{-1}$ of $M_{\mathrm{L}}$ is also smaller than their value $134 \AA^{-1}$, the former value being consistent with that estimated on the basis of the chain conformations of PDiPF. It may then be said that analyses of data for PDiPF in $\mathrm{THF}$ at $30.0^{\circ} \mathrm{C}$ without consideration of the excluded-volume effects lead to overestimation of both the values of $\lambda^{-1}$ and $M_{\mathrm{L}}$.

In order to confirm the result of the present analysis of $\left\langle S^{2}\right\rangle$ and $A_{2}$, it is desirable to make a study of steady-state transport coefficients such as $[\eta]$ and the translational diffusion coefficient of PDiPF. Thus we proceed to make such a study in a forthcoming paper.

Received: January 25, 2008

Accepted: March 13, 2008

Published: May 2, 2008

\section{REFERENCES}

1. W. I. Bengough, G. B. Park, and R. A. Young, Eur. Polym. J., 11, 305 (1975).

2. T. Otsu, O. Ito, N. Toyoda, and S. Mori, Makromol. Chem., Rapid Commun., 2, 725 (1981).

3. T. Otsu, T. Yasuhara, K. Shiraishi, and S. Mori, Polym. Bull., 12, 449 (1984).

4. T. Otsu, H. Minai, N. Toyoda, and T. Yasuhara, Makromol. Chem., Suppl., 12, 133 (1985).

5. T. Otsu, T. Yasuhara, and A. Matsumoto, J. Macromol. Sci. Chem., A25, 537 (1988).
6. A. Matsumoto and E. Nakagawa, Eur. Polym. J., 35, 2107 (1999).

7. O. Kratky and G. Porod, Recl. Trav. Chim. Pays-Bas, 68, 1106 (1949).

8. H. Yamakawa, "Helical Wormlike Chains in Polymer Solutions," Springer, Berlin, 1997.

9. F. Abe, Y. Einaga, T. Yoshizaki, and H. Yamakawa, Macromolecules, 26, 1884 (1993).

10. Y. Tamai, T. Konishi, Y. Einaga, M. Fujii, and H. Yamakawa, Macromolecules, 23, 4067 (1990).

11. H. Murakami, T. Norisuye, and H. Fujita, Macromolecules, 13, 345 (1980).

12. F. Kasabo, T. Kanematsu, T. Nakagawa, T. Sato, and A. Teramoto, Macromolecules, 33, 2748 (2000).

13. H. Yamakawa and W. H. Stockmayer, J. Chem. Phys., 57, 2843 (1972).

14. H. Yamakawa and J. Shimada, J. Chem. Phys., 83, 2607 (1985).

15. J. Shimada and H. Yamakawa, J. Chem. Phys., 85, 591 (1986).

16. H. Yamakawa, Macromolecules, 25, 1912 (1992).

17. Gj. Deželić and J. Vavra, Croat. Chem. Acta, 38, 35 (1966).

18. G. C. Berry, J. Chem. Phys., 44, 4550 (1966).

19. H. Yamakawa, "Modern Theory of Polymer Solutions," Harper \& Row, New York, 1971. Its electronic edition is available on-line at the URL: http://www.molsci.polym.kyoto-u.ac.jp/archives/redbook.pdf

20. H. Utiyama and M. Kurata, Bull. Inst. Chem. Res. Kyoto Univ., 42, 128 (1964).

21. H. Utiyama, J. Phys. Chem., 69, 4138 (1965).

22. K. Nagai, Polym. J., 3, 67 (1972).

23. H. Yamakawa, M. Fujii, and J. Shimada, J. Chem. Phys., 71, 1611 (1979).

24. X. Wang, T. Komoto, I. Ando, and T. Otsu, Makromol. Chem., 189, 1845 (1988).

25. M. Yoshioka, A. Matsumoto, and T. Otsu, Polym. J., 23, 1191 (1991).

26. H. Benoit and P. Doty, J. Phys. Chem., 57, 958 (1953).

27. C. Domb and A. J. Barrett, Polymer, 17, 179 (1976).

28. Y. Einaga, F. Abe, and H. Yamakawa, Macromolecules, 26, 6243 (1993).

29. F. Abe, Y. Einaga, and H. Yamakawa, Macromolecules, 27, 3262 (1994).

30. M. Kamijo, F. Abe, Y. Einaga, and H. Yamakawa, Macromolecules, 28, 4159 (1995).

31. W. Tokuhara, M. Osa, T. Yoshizaki, and H. Yamakawa, Macromolecules, 36, 5311 (2003). 